\title{
Culture Qualitatively but Not Quantitatively Influences Performance in the Boston Naming Test in a Chinese-Speaking Population
}

\author{
Ting-Bin Chen ${ }^{a}$ b $\quad$ Chi-Ying Lin ${ }^{a}, b \quad$ Ker-Neng Lin ${ }^{a, d} \quad$ Yen-Chi Yeh ${ }^{b}, f$ \\ Wei-Ta Chen ${ }^{a, b}$ Kuo-Shu Wang ${ }^{\mathrm{e}}$ Pei-Ning Wang ${ }^{\mathrm{a}-\mathrm{c}}$ \\ a Department of Neurology, Neurological Institute, Taipei Veterans General Hospital, \\ ${ }^{b}$ Department of Neurology, National Yang-Ming University School of Medicine, ${ }^{\mathrm{c}}$ Aging \\ and Health Research Center, National Yang-Ming University, ${ }^{d}$ Department of Psychology, \\ Soochow University, and ${ }^{\mathrm{G}} \mathrm{Graduate}$ Institute of Linguistics, National Taiwan University, \\ Taipei, and ${ }^{\mathrm{f}}$ Department of Neurology, Kuo General Hospital, Tainan, Taiwan (ROC)
}

Key Words

Boston Naming Test · Chinese-speaking population - Cross-cultural comparison · Elderly · Taiwan

\begin{abstract}
Background/Aims: The Boston Naming Test (BNT) is the most frequently administered confrontational naming test, but the cultural background of the patients may influence their performance in the BNT. The aim of this study was to identify differences in performance in the BNT between a Chinese population in Taiwan, Chinese populations in other areas and a Caucasian population. Methods: A total of 264 native, Chinese-speaking, cognitively normal elders aged $>60$ years were enrolled in our study and conducted the 30 -item Chinese version of the BNT. Another 10 BNT studies were categorized, analyzed and compared with the present study. Results: Higher education was associated with higher scores, whereas age and gender had no effect on performance in the BNT. The score of the Chinese-speaking population was equivalent to the English-speaking population. A disparity in difficulties with items was not only apparent between the Taiwanese and Caucasian populations, but also between the Chinese-speaking populations in the different geographic areas. Conclusion: For the most part, the impact of culture on performance in the BNT may not be quantitative but qualitative. Attention should be paid to a potential effect of culture on difficulties with items when administering the BNT to non-English-speaking populations. Understanding differences in performance in the BNT in distinct cultural settings improves the clinical application of the BNT.




\section{Introduction}

Word finding difficulty is a common complaint in the elderly. It may be a phenomenon deriving from the effects of aging on cognition, but could also be one of the early signs of dementia. Therefore, using an appropriate neuropsychological test to assess the naming ability in elders is important in screening possible cognitive impairment in this population.

The Boston Naming Test (BNT) [1], a visual confrontational naming task consisting of 60 outline drawings of objects and animals, is the most frequently administered confrontational naming test. The BNT has been validated for identifying naming deficits in a variety of neurological diseases with different pathophysiologies, such as cerebrovascular accidents, traumatic brain injury and neurodegenerative diseases. In elders in their 70s and 80s, word finding difficulty can be detected by their performance in the BNT as well.

In spite of its clinical utility, the content of the BNT reflects the cultural context in which it was developed. The items in the BNT are constructed in the order of word frequency and grades of difficulty. Although the BNT has been available for many populations with different linguistic backgrounds, many investigators have concerns about cultural relevance, as word frequency and participants' familiarity with test items differ among populations, races and countries. Performance in the BNT may be underestimated and misinterpreted outside the USA. Therefore, it is imperative and important to establish norms for different cultural and linguistic populations.

Of the different language versions of the BNT, some adopt the original English items [2, $3]$ and others make adjustments to some items according to their cultural background $[4,5]$. There are several Chinese versions of the BNT [6-8], all of which adopted the original English items [1] and showed that it is practical to adopt the BNT for Chinese populations for the evaluation of dementia, radiotherapy-related brain injury and temporal lobe epilepsy with different pathologies [6-8]. However, there has been no study so far designed to make a comparison of performance in the BNT in a normal elderly Chinese population between different ethnic groups.

The 30-item BNT used in our study was developed for and applied to a Chinese population in Hong Kong (HK) [8]. The authors successfully demonstrated its validity for detecting naming impairment in head injury patients in comparison with a normal control group. This Chinese version of the BNT used 30 items selected from the original English version, but according to a Chinese cultural background. The advantage of a BNT without item adjustment and replacement lies in making comparisons of performance in the BNT between Chinese and Caucasian populations simpler and easier. Meanwhile, it also provides an opportunity to examine differences in the effect of Chinese culture on performance in the BNT between Chinese populations inhabiting different geographical regions.

We administered this Chinese version of the BNT to a Chinese elderly population in Taiwan (TW) to establish norms for this test in Taiwanese elders. The effect of demographic variables, including education, age and gender, on naming performance was examined. Our data on performance in the BNT were compared with Chinese populations in other areas and with Caucasian populations with different linguistic backgrounds in order to investigate the impact of culture on the BNT.

\section{Subjects and Methods}

This study was conducted from January 2006 to November 2007 at Taipei Veterans General Hospital. The local institutional review board approved data collection for this study. Written informed consent was obtained from all participants. 
Chen et al.: Culture Qualitatively but Not Quantitatively Influences Performance in the Boston Naming Test in a Chinese-Speaking Population

\section{Subjects}

Participants were recruited from volunteers or spouses or caregivers of patients in memory clinics. Neurologists interviewed all the subjects. A diagnosis of normal was based on the results of the clinical interviews, neurological examinations, neuropsychological tests, laboratory findings and neuroimaging evaluation. Any individuals with a history of psychiatric or neurological diseases, stroke, head trauma, present or past abuse or daily use of alcohol or learning disability that was likely to have an influence on performance in the BNT were excluded.

Trained assistants administered the 30-item BNT [8] and Mini-Mental State Examination (MMSE) [9]. Each subject and a family member or primary caregiver were interviewed independently by a neuropsychologist or neurologist using the Clinical Dementia Rating [10]. The normal subjects recruited for this study were required not to have subjective complaints of significant memory decline. All of them had an MMSE score of $\geq 24$ and a Clinical Dementia Rating score of 0 .

\section{Chinese Version of the 30-Item BNT}

The 30-item BNT used in this study is adapted from the modified version previously developed and applied to a Chinese population in HK [8]. These 30 items were selected from the original 60 items based on the cultural relevance of the items in the local context. The order of presentation of the stimuli followed the original sequence, and the design of the stimulus cards was identical to that of the original pictures. This version had demonstrated good validity for detecting naming impairment in Cantonese-speaking head injury patients in HK.

\section{Procedures and Scoring}

The participants were told to tell the examiner the name of each object depicted. All pictures were identical to those in the original BNT. They were required to complete all 30 items. Up to $20 \mathrm{~s}$ were allowed to name each item. The Chinese name of the object on the stimulus card was set as the target response. If a participant named the item correctly, the examiner proceeded to the next item, credits were given for self-corrections and it was recorded among 'scores of spontaneous naming (SN)'. If a participant gave a wrong response, indicated that he or she did not know the answer, or gave no response within $20 \mathrm{~s}$, the examiner wrote down the patient's response in detail and a standard semantic cue was provided (e.g. 'it is a tool' for 'saw'). A semantic cue was designed for each response as in the original version of the BNT. If a subject gave the correct answer, credits were given and recorded as 'scores after semantic cue'. On the other hand, if a subject could not name the object correctly after a semantic cue had been provided, a standard phonemic cue was given. The phonemic cue was given according to the following rules: (1) if the name of the item was a single Chinese character, then the initial phoneme of the target answer was given, and (2) if the name of the item had multiple Chinese characters, the pronunciation of the first word was provided. If the participant gave the correct response after the phonemic cue, credits were given and it was recorded among 'scores after phonemic cue'.

The number of correct answers after semantic or phonemic cues was divided by the number of all nontarget answers and recorded as the 'ratio of correct answers after semantic cues or phonemic cues'. Scores of total correct naming (TN) were defined as scores of SN plus scores after semantic and phonemic cues.

\section{Statistical Analysis}

Descriptive statistics for demographic and neuropsychological data are presented as means \pm standard deviation. Statistical analysis was performed by SPSS version 17.0 for 
Chen et al:: Culture Qualitatively but Not Quantitatively
Boston Naming Test in a Chinese-Speaking Population

Windows (SPSS Inc., Chicago, Ill., USA). The effect of gender on BNT scores was analyzed by independent-sample $t$ test. Effects of group differences in educational level on performance in the BNT were evaluated by one-way analysis of variance with Tukey's post hoc test for multiple comparisons. Pearson's correlation coefficients were used to determine the significance and strength of the relationship between BNT score and demographic variables. The percentage of correct responses on SN for each item was calculated and yielded an index of difficulty level. For all tests, $\mathrm{p}<0.05$ was considered statistically significant.

\section{Cross-Cultural Comparison of Performance in the BNT}

BNT studies carried out in the USA [11], Canada (CA) [2], Quebec French-speaking Canada (QF) [12], Australia (AU) [13], New Zealand (NZ) [14], Sweden (SW) [3], Spain (SP) [15], Greece (GR) [4], Belgium (BE) [16] and HK [8] were reviewed and compared with the present study according to differences in the percentages of correct responses to each item. A percent difference per item of $>20 \%$ compared with our item percentage was considered significant.

\section{Results}

In total, 264 cognitively normal elders, 156 men (59.0\%) and 108 women (40.9\%), were recruited for the current study. The age range was 60-92 years, with an average of 73.4 years. The average period of formal education was 12.5 years (range: 0-19 years). MMSE scores ranged between 24 and 30, with an average of 28.2 .

\section{Internal Consistency of the Chinese Version of the BNT}

The 30 items composing the Chinese version of the BNT showed a high internal reliability coefficient $(\alpha=0.85)$, suggesting that the items of this test reliably measure the same construct. Every item comprised in the BNT was positively correlated with the total score and contributed positively to Cronbach's $\alpha$ for the total score. The overall mean scores of SN and TN were 24.7 \pm 3.9 and $27.6 \pm 2.4$. The ratios of correct answers after semantic cues or phonemic cues were $0.1 \pm 0.2$ and $0.3 \pm 0.2$, respectively.

\section{Effect of Education on Performance in the BNT}

Correlation analysis demonstrated a positive correlation between years of education and BNT score $(\mathrm{r}=0.376, \mathrm{p}<0.01)$. Therefore, the subjects were subgrouped into four educational categories: $(1)<6$ years $(n=14)$; (2) 6-8 years $(n=22)$; (3) 9-12 years $(n=93)$, and $(4)>12$ years $(n=135)$. According to the post hoc analysis, a between-group difference mainly occurred between 6-8 years and 9-12 years of education in scores of SN ( $22.0 \pm 3.7$ vs. 25.1 $\pm 3.0 ; \mathrm{p}<0.01)$ and TN $(25.5 \pm 3.1$ vs. $27.8 \pm 1.6 ; \mathrm{p}<0.01)$. There were no significant differences between $<6$ years and $6-8$ years of education and between $9-12$ years and $>12$ years of education in scores for SN (<6 vs. $6-8$ years: $20.6 \pm 4.5$ vs. $22.0 \pm 3.7 ; 9-12$ vs. $>12$ years: $25.1 \pm 3.0$ vs. $25.7 \pm 4.0$ ) and TN (<6 vs. $6-8$ years: $24.5 \pm 4.1$ vs. $25.5 \pm 3.1 ; 9-12$ vs. $>12$ years: $27.8 \pm 1.6$ vs. $28.1 \pm 2.1$ ). Therefore, we redivided the subjects into two education groups $(<9$ and $\geq 9$ years). Table 1 presents the BNT scores for the two education groups.

\section{Effect of Sex on Performance in the BNT}

In general, performance in the BNT was not significantly different between men and women with regard to any of the scores (table 2). However, if we focused on the subjects with higher education ( $\geq 9$ years), women performed better than men in SN (men vs. women: $24.9 \pm 4.1$ vs. $25.6 \pm 2.6 ; p=0.02$ ) and in TN (men vs. women: $27.9 \pm 2.2$ vs. $28.1 \pm 1.4 ; p=0.02$ ). Performance in the BNT was not different between men and women in the group with lower education. 
Table 1. Characteristics and MMSE and BNT results according to two different educational levels and for all subjects

\begin{tabular}{l|l}
\hline Dement Geriatr Cogn Disord Extra 2014;4:86-94 \\
\hline DOI: 10.1159/000360695 & $\begin{array}{l}\text { @ 2014 S. Karger AG, Basel } \\
\text { www.karger.com/dee }\end{array}$ \\
\hline
\end{tabular}

Chen et al.: Culture Qualitatively but Not Quantitatively Influences Performance in the Boston Naming Test in a Chinese-Speaking Population

\begin{tabular}{lccc}
\hline & \multicolumn{2}{c}{ Education } & \multirow{2}{*}{ Total } \\
\cline { 2 - 3 } & $<9$ years & $\geq 9$ years & \\
\hline Number & 36 & 228 & 264 \\
Males, \% & 30.5 & 63.5 & 59.0 \\
MMSE score & $27.7 \pm 1.5$ & $28.0 \pm 1.9$ & $28.2 \pm 1.4$ \\
BNT score & & & \\
$\quad$ SN & $21.5 \pm 4.0$ & $25.2 \pm 3.6$ & $24.7 \pm 3.9$ \\
SC & $1.1 \pm 1.1$ & $0.8 \pm 1.1$ & $0.9 \pm 1.1$ \\
RSC & $0.1 \pm 0.1$ & $0.1 \pm 0.2$ & $0.1 \pm 0.2$ \\
PC & $2.5 \pm 1.9$ & $1.7 \pm 1.6$ & $1.8 \pm 1.6$ \\
RPC & $0.3 \pm 0.1^{*}$ & $0.3 \pm 0.2^{*}$ & $0.3 \pm 0.2$ \\
TN & $25.1 \pm 3.5^{* *}$ & $28.0 \pm 1.9^{* *}$ & $27.6 \pm 2.4$ \\
\hline
\end{tabular}

Data are presented as means \pm standard deviations unless specified otherwise, and analyzed by independent-sample t test. PC $=$ Score after phonemic cue; $\mathrm{RPC}=$ ratio of correct answers after phonemic cue; $\mathrm{RSC}=$ ratio of correct answers after semantic cue; $\mathrm{SC}=$ score after

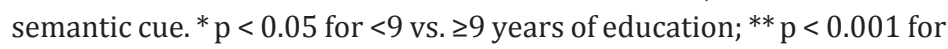
$<9$ vs. $\geq 9$ years of education.

Table 2. BNT results according to gender

\begin{tabular}{|c|c|c|c|c|c|c|}
\hline & \multicolumn{3}{|c|}{ Education (males) } & \multicolumn{3}{|c|}{ Education (females) } \\
\hline & $<9$ years & $\geq 9$ years & total & $<9$ years & $\geq 9$ years & total \\
\hline Number & 11 & 145 & 156 & 25 & 83 & 108 \\
\hline \multicolumn{7}{|l|}{ BNT score } \\
\hline $\mathrm{SN}$ & $22.7 \pm 4.1$ & $24.9 \pm 4.1^{*}$ & $24.8 \pm 4.1$ & $20.9 \pm 4.0$ & $25.6 \pm 2.6^{*}$ & $24.5 \pm 3.5$ \\
\hline SC & $1.1 \pm 1.1$ & $0.9 \pm 1.1$ & $0.9 \pm 1.1$ & $1.1 \pm 1.1$ & $0.7 \pm 0.9$ & $0.8 \pm 0.9$ \\
\hline RSC & $0.2 \pm 0.1$ & $0.1 \pm 0.1$ & $0.1 \pm 0.2$ & $0.1 \pm 0.1$ & $0.1 \pm 0.2$ & $0.1 \pm 0.2$ \\
\hline PC & $2.2 \pm 1.4$ & $1.8 \pm 1.6$ & $1.8 \pm 1.5$ & $2.6 \pm 2.1$ & $1.7 \pm 1.6$ & $1.9 \pm 1.8$ \\
\hline $\mathrm{RPC}$ & $0.3 \pm 0.2$ & $0.3 \pm 0.2$ & $0.3 \pm 0.2$ & $0.3 \pm 0.1$ & $0.3 \pm 0.2$ & $0.3 \pm 0.2$ \\
\hline $\mathrm{TN}$ & $26.1 \pm 3.3$ & $27.9 \pm 2.2^{*}$ & $27.8 \pm 2.3$ & $24.7 \pm 3.6$ & $28.1 \pm 1.4^{*}$ & $27.3 \pm 2.5$ \\
\hline
\end{tabular}

Data are presented as means \pm standard deviations unless specified otherwise, and analyzed by independent-sample t test. $\mathrm{PC}=$ Score after phonemic cue; RPC = ratio of correct answers after phonemic cue; $\mathrm{RSC}=$ ratio of correct answers after semantic cue; $\mathrm{SC}=$ score after semantic cue. ${ }^{*} \mathrm{p}<0.05$ for males vs. females.

\section{Effect of Age on Performance in the BNT}

There was no significant correlation between age and any of the BNT scores by Pearson's correlation analysis. The subjects were subsequently divided into three age groups (60-69, 70-79 and $\geq 80$ years), but there still was no significant difference between these groups.

\section{Percentage of Responses in the BNT}

The overall accuracy of the participants' responses was $92.1 \%(7,293$ of the 7,920 items were correct) in our study. Table 3 lists the items in their order of difficulty (percent correct). There were 2 items, 'pencil' and 'scissors', showing 100\% correct responses. 'Protractor' 
Chen et al.: Culture Qualitatively but Not Quantitatively Influences Performance in the Boston Naming Test in a Chinese-Speaking Population

Table 3. Accuracy of responses per item in our study and 10 published studies on the BNT performed in healthy adult populations

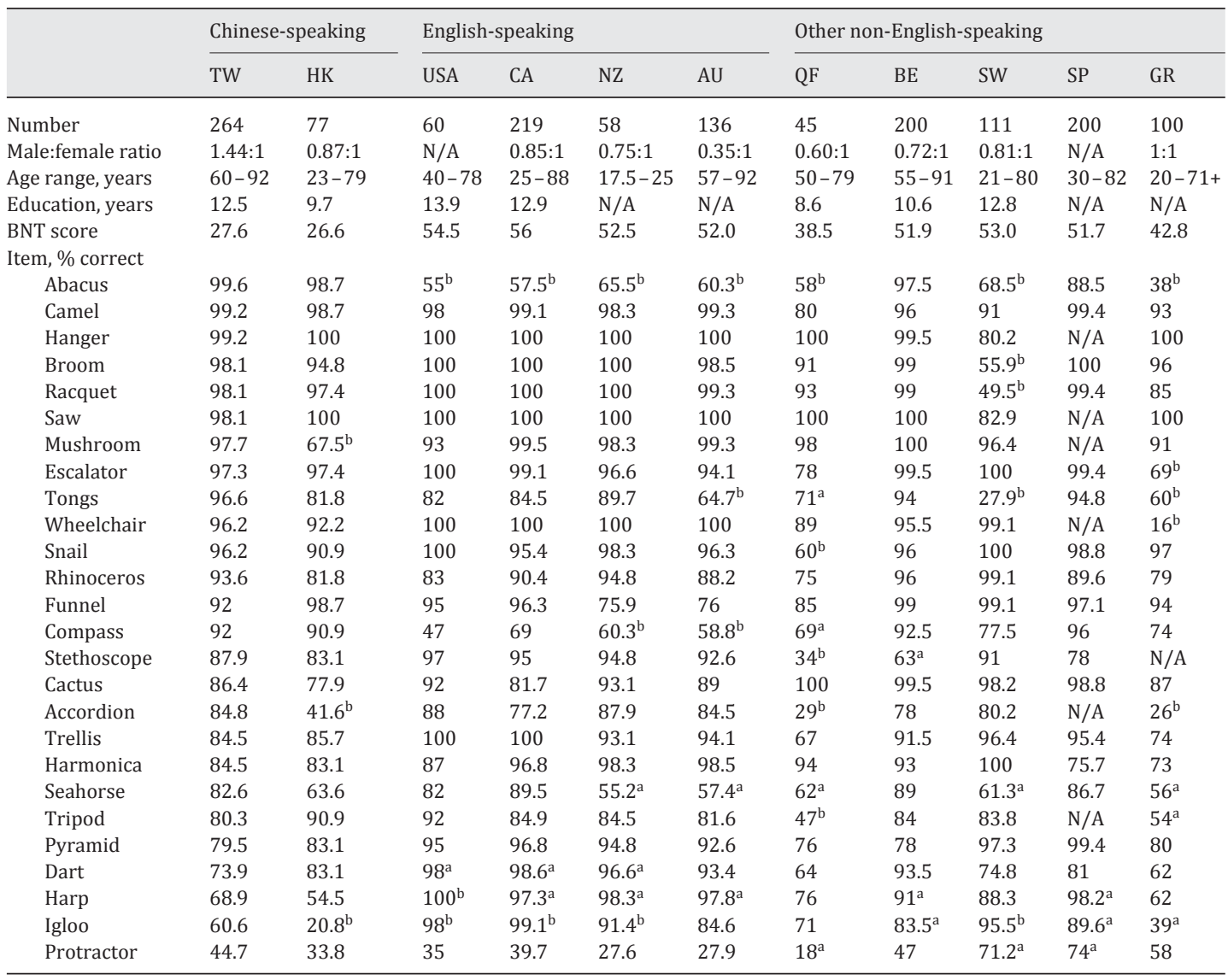

Values for education and BNT are means. N/A = Not available.

${ }^{a}>20 \%$ difference as compared with our data. ${ }^{b}>30 \%$ difference as compared with our data.

displayed the fewest correct responses (44.7\%). The participants with low education made $20 \%$ more errors than the highly educated subjects on 'stethoscope', 'accordion', 'trellis', 'harmonica', 'tripod', 'pyramid', 'dart', 'harp' and 'protractor'.

\section{Cross-Cultural Comparison of BNT Performance}

All 11 BNT studies were classified into three groups: Chinese-speaking, English-speaking and non-English-speaking countries. TW and HK used the same 30-item BNT, while the others administered the 60 -item BNT. In TW and HK, the total scores were within a similar range (TW: $27.6 \pm 2.4$; HK: $26.6 \pm 2.75$ ); in the English-speaking populations they were around 55 (USA: $54.5 \pm 3.5$; CA: $58.3 \pm 2.0$; NZ: $52.5 \pm 2.2$; AU: $52.0 \pm 6.3$ ), and in the non-English-speaking countries they showed a wide range (QF: $38.5 \pm 7.2$; SW: $53.0 \pm 4.1$; SP: $51.7 \pm 4.7$; GR: $42.8 \pm 9.7$; BE: $51.9 \pm 5.5$ ). As a whole, compared with the TW study, the number of items showing a significant difference $(>20 \%)$ in the percentage of correct responses was 3 for HK (mushroom, accordion and igloo), 7 for the English-speaking participants (abacus, tongs, compass, seahorse, dart, harp and igloo) and 16 for the non-English-speaking subjects (tree, abacus, broom, racquet, escalator, tongs, wheelchair, snail, compass, stethoscope, accordion, seahorse, tripod, harp, igloo and protractor). 'Abacus' was nearly invariably difficult for non-Chinese-speaking populations; by contrast, 'igloo' was almost consistently easy for English-speaking populations. 
Chen et al.: Culture Qualitatively but Not Quantitatively Influences Performance in the Boston Naming Test in a Chinese-Speaking Population

\section{Discussion}

The BNT was developed for English-speaking populations. The psychometrics of the BNT has been rarely reported on and evaluated in a Chinese population. We found educational level to be positively correlated with performance in the BNT. The total BNT scores were numerically close in populations with different languages and cultural backgrounds. Nevertheless, the rates for accuracy of response per item and the order of items according to difficulty varied. This is in accord with a qualitative, rather than a quantitative, influence on performance in the BNT.

\section{Effect of Demographics on Performance in the BNT}

In the elderly, education showed a significant effect on performance in the BNT, but age and gender did not. Radanovic et al. [17] indicated that 9 years of education are necessary to gain the knowledge of lexical items frequently acquired in daily life. Our study supported that a 9-year education is essential for establishing adequate naming abilities.

The impact of age on cognitive performance is often exaggerated by cohort effects in cross-sectional studies $[18,19]$. A prospective study has demonstrated that confrontational naming ability is generally well preserved in aging, with only a subtle decline in the 7th and 8th decades of life [20]. Our data suggest that age is not an important factor influencing performance in the BNT for subjects aged $>60$ years.

\section{Cultural Differences in Performance in the BNT}

The BNT was originally designed to be administered to English-speaking North American populations. Due to its value and accessibility, there has been an increasing number of translations and adaptations of this test for use in a variety of populations with different languages and cultures. The study from NZ [14] only enrolled young participants aged between 17.5 and 25 years, but the other 10 studies [this study; 2-4, 8, 11-13, 15, 16] all included elderly subjects aged $>60$ years. The studies from SP [15] and GR [4] replaced several items in the 60 -item BNT based on their cultural background. As mentioned below, an analysis of item frequencies across different cultural backgrounds was made by direct comparison of 30 items selected from all studies.

\section{Differences between TW and English-Speaking Countries}

Hobson et al. [21] showed a reliable creation of an estimated 60-item BNT score from administrations of the 30 -item BNT by multiplying the obtained score by 2 . By this method, the total BNT scores from our study and the one from HK were multiplied by 2 to allow comparisons across different studies more easily and simply. The calculated total BNT scores for elders in TW (estimated BNT score of 55) and HK (estimated BNT score of 53) were close to those in the English-speaking populations. Although the total BNT scores were similar between populations with different cultural backgrounds, the accuracy rates for each item varied. The most significant difference in item difficulty was that our subjects performed better on the item 'abacus'. The abacus is a familiar tool for calculation used in parts of Asia, and many Chinese elders used it in their early lives. However, our subjects performed worse on 'pyramid', 'dart', 'harp' and 'igloo', which are uncommon and unfamiliar to elders in TW. This diversity could be attributed to the accessibility of test objects in everyday life in distinct cultures.

\section{Differences between TW and HK}

Inhabitants of TW and HK are culturally similar and live geographically close to each other. Although traditional Mandarin Chinese is used as the common language of writing, 
people in TW speak Mandarin and Taiwanese and people in HK speak Cantonese, Mandarin and English. It would be expected that BNT results differ between respondents from TW and HK as they possess varying spoken-language skills. Besides, in daily adult-to-adult conversation, word frequencies are different in the two locations. These linguistic differences lead to the effect that certain words are more or less prominent, or there is familiarity with some words more than with others, and this may inevitably cause a bias in language-oriented neuropsychological instruments [22]. Therefore, cultural-linguistic and related differences between TW and HK may affect performance in neuropsychological tests. The total BNT scores were similar between the two populations. Although the inhabitants of both HK and TW used Chinese characters for written communication, the participants in TW made fewer errors than the HK sample regarding 3 items (i.e. mushroom, trellis and igloo). The inconsistency in performance in individual BNT items between these two populations may result from dialect use and Western educational policy in HK.

The same geographic, cultural and linguistic relationship as found between TW and HK could also be observed between CA and QF; residents of QF performed much worse than subjects in CA on 10 items (i.e. escalator, snail, trellis, seahorse, tripod, pyramid, dart, harp, igloo and protractor).

\section{Differences between English-Speaking and Non-English-Speaking Countries}

The non-English-speaking countries showed varied total BNT scores. GR and QF scored lower on the BNT than other countries. Patricacou et al. [4] pointed out that the poorer performance in GR may be attributed to the high proportion (25\%) of participants with a low education level ( $<6$ years of education) and the high percentage of older examinees $(42 \%$ were $>60$ years and 20\% $>70$ years of age). Roberts and Doucet [12] demonstrated that the mean BNT scores were similar between QF and English-speaking Americans with similarly low levels of education. Nevertheless, the non-English-speaking participants revealed better performance on the items 'compass', 'abacus' and 'protractor', but had relatively inferior outcomes on the items 'tongs', 'cactus', 'trellis', 'tripod', 'dart', 'harp' and 'igloo'. On the whole, better recognition of items by English speakers implied a greater familiarity and acquaintance with the test objects in English-speaking countries and vice versa.

\section{Limitations}

There are some limitations to our study. First, the participants enrolled in our study were mainly elders aged $>60$ years, so the data may not be generalized to adults of all ages. Second, the data should be only cautiously applied to elderly people aged $>80$ years because of the relatively small size of the oldest-old group in this study. Third, most of our participants were highly educated and there were only few illiterate subjects. The effect of education on performance in the BNT may be underestimated. Fourth, all studies discussed in this manuscript were conducted in various decades; 5 of them were carried out before the year 2000, and cohort effects may have contributed to differences in performance in the BNT.

The 30 items examined in our study and that from HK were adapted from the original version of the 60 -item BNT. Therefore, cultural influences on all 60 items were not thoroughly analyzed in these two studies. Besides, the analysis of the total scores among all populations was conducted by a comparison between the estimated 60-item BNT scores calculated from the 30 -item test by means of multiplication by 2 in our and the HK study and the 60 -item BNT scores in the other studies. By utilizing the same version of the BNT in our and the HK study, it was observable that different geographic backgrounds did lead to cultural variation. 
Chen et al.: Culture Qualitatively but Not Quantitatively Influences Performance in the Boston Naming Test in a Chinese-Speaking Population

\section{Conclusion}

The Chinese version of the 30-item BNT demonstrated acceptable applicability to elders in TW. Education is the major demographic factor and may influence performance in the BNT. Chinese-speaking populations, as in TW and HK, scored equivalently on the BNT compared with English-speaking populations. However, the responses to and difficulties with individual items of the BNT may vary in populations with different languages and cultural backgrounds.

\section{References}

1 Goodglass H, Kaplan E: The Assessment of Aphasia and Related Disorders, ed 2. Philadelphia, Lea \& Febiger, 1983.

-2 Tombaugh TN, Hubley AM: The 60-item Boston Naming Test: norms for cognitively intact adults aged 25 to 88 years. J Clin Exp Neuropsychol 1997;19:922-932.

3 Tallberg IM: The Boston Naming Test in Swedish: normative data. Brain Lang 2005;94:19-31.

4 Patricacou A, Psallida E, Pring T, Dipper L: The Boston Naming Test in Greek: normative data and the effects of age and education on naming. Aphasiology 2007;21:1157-1170.

-5 Peña-Casanova J, Quiñones-Ubeda S, Gramunt-Fombuena N, et al: Spanish Multicenter Normative Studies (NEURONORMA Project): norms for Boston naming test and Token test. Arch Clin Neuropsychol 2009;24: 343-354.

-6 Salmon DP, Jin H, Zhang M, Grant I, Yu E: Neuropsychological assessment of Chinese elderly in the Shanghai Dementia Survey. Clin Neuropsychol 1995;9:159-168.

7 Cheung M, Chan AS, Law SC, Chan JH, Tse VK: Cognitive function of patients with nasopharyngeal carcinoma with and without temporal lobe radionecrosis. Arch Neurol 2000;57:1347-1352.

8 Cheung RW, Cheung MC, Chan AS: Confrontation naming in Chinese patients with left, right or bilateral brain damage. J Int Neuropsychol Soc 2004;10:46-53.

-9 Folstein MF, Folstein SE, McHugh PR: 'Mini-Mental State': a practical method for grading the cognitive state of patients for the clinician. J Psychiatr Res 1975;12:189-198.

10 Hughes CP, Berg L, Danziger WL, Coben LA, Martin RL: A new clinical scale for the staging of dementia. Br J Psychiatry 1982;140:566-572.

11 Nicholas L, Brookshire R, McLennan D, Schumacher J, Porrazzo S: Revised administration and scoring procedures for the Boston Naming Test and norms for non-brain-damaged adults. Aphasiology 1989;3:569-580.

12 Roberts PM, Doucet N: Performance of French-speaking Quebec adults on the Boston Naming Test. Can J Speech Lang Pathol Audiol 2011;35:254-267.

13 Worrall LE, Yiu EML, Hickson LMH, Barnett HM: Normative data for the Boston Naming Test for Australian elderly. Aphasiology 1995;9:541-551.

14 Barker-Collo SL: The 60-item Boston Naming Test: cultural bias and possible adaptations for New Zealand. Aphasiology 2001;15:85-92.

-15 Allegri RF, Mangone CA, Fernandez-Villavicencio A, Rymberg S, Taragano FE, Baumann D: Spanish Boston Naming Test norms. Clin Neuropsychol 1997;11:416-420.

16 Mariën P, Mampaey E, Vervaet A, Saerens J, de Deyn PP: Normative data for the Boston Naming Test in native Dutch-speaking Belgian elderly. Brain Lang 1998;65:447-467.

17 Radanovic M, Mansur LL, Scaff M: Normative data for the Brazilian population in the Boston Diagnostic Aphasia Examination: influence of schooling. Braz J Med Biol Res 2004;37:1731-1738.

18 Schaie KW, Hofer SM: Longitudinal studies in research on aging; in Birren JE, Schaie KW (eds): Handbook of the Psychology of Aging, ed 5. San Diego, Academic Press, 2001.

19 Schaie KW: The impact of longitudinal studies on understanding development from young adulthood to old age. Int J Behav Dev 2002;24:257-266.

20 Zec RF, Markwell SJ, Burkett NR, Larsen DL: A longitudinal study of confrontation naming in the 'normal' elderly. J Int Neuropsychol Soc 2005;11:716-726.

21 Hobson VL, Hall JR, Harvey M, Cullum CM, Lacritz L, Massman PJ, Waring SC, O’Bryant SE: An examination of the Boston Naming Test: calculation of 'estimated' 60-item score from 30- and 15-item scores in a cognitively impaired population. Int J Geriatr Psychiatry 2011;26:351-355.

22 Marquez de la Plata C, Arango-Lasprilla JC, Alegret M, Moreno A, Tárraga L, Lara M, Hewlitt M, Hynan L, Cullum CM: Item analysis of three Spanish naming tests: a cross-cultural investigation. NeuroRehabilitation 2009;24: 75-85. 


\section{Erratum}

In the article by Chen et al., entitled 'Culture qualitatively but not quantitatively influences performance in the Boston Naming Test in a Chinese-speaking population' [Dement Geriatr Cogn Disord Extra 2014;4:86-94, DOI: 10.1159/000360695], the following acknowledgments were erroneously left out:

\section{Acknowledgment}

This work was supported in part by the National Science Council (NSC 101-2314-B-010-064, NSC 102-2314-B-010-051-MY2) and the Taipei Veterans General Hospital (V96C1-061, V101E7-001, V102E9004). The authors also acknowledge MR support from the Ministry of Education of Taiwan (Aim for the Top University Plan). 